\title{
RFID and PIR Motion Sensor based Automated Attendance System for Educational Institutions
}

\author{
K. Srividhyasaradha, I. Joe Louis Paul, S. Sasirekha
}

\begin{abstract}
Automated attendance system is the advancement that has taken place in the field of automation replacing traditional attendance marking activities. Designing an automated attendance system provides full transparency and maintains a regular and accurate updating of the attendance in the database. This system makes the task of attendance management easier while allowing correct attendance status and making the same data available to all concerned. This is implemented by integrating two technologies to achieve a foolproof system for effective attendance marking. This can be made feasible by synchronizing a combination of Radio Frequency Identification Detection (RFID) and Passive Infrared (PIR) motion sensor. Based on the verification the student identity, the RFID tag can be embedded in the ID card of the individual and their presence can be detected by a PIR motion sensor
\end{abstract}

Keywords: RFID, PIR Motion Sensor, Smart Card, Automated Attendance System, Android Application

\section{INTRODUCTION}

Automation of attendance system is an important part of any organization. Every academic institution has certain criteria for students regarding their attendance in the classroom. Proper participation in classroom inculcates a sense of strong training environment in the minds of students. Monitoring the attendance of the students is needed to ensure healthy learning atmosphere.

A significant amount of time is wasted for both the staff and students while marking the attendance manually. Moreover, it is a tedious task to take the attendance too. There are additional malpractices like proxy which makes the system further flawed. There are other downsides like misplacement of attendance sheet and students cannot access the information about their attendance. There are further chances that can make the loss of such attendance sheets lead to an increase in the students marking their fake presence.

One solution proposed to this method was to record the attendance in Excel sheets [1] and regularly send updates to parents via Short Messaging Services (SMS). This would ensure parents regularly keep track of the wards and also ensure better classroom participation. But this method was not very feasible as it incurs additional time. In addition, the attendance is recorded manually by the tutor and therefore may be prone to personal errors. The whole process is highly cumbersome in nature. The manual method of taking attendance is a very time consuming, inefficient and

Revised Version Manuscript Received on August 19, 2019.

K. Srividhyasaradha, Undergraduate Student, Department of IT, SSN College of Engineering, Kalavakkam, Tamil Nadu, India.

I. Joe Louis Paul, Associate Professor, Department of IT, SSN College of Engineering, Kalavakkam, Tamil Nadu, India (Email: joelouisi@ssn.edu.in)

S. Sasirekha, Associate Professor, Department of IT, SSN College of Engineering, Kalavakkam, Tamil Nadu, India monotonous activity. To overcome the drawbacks associated with the traditional method of attendance marking, a barcode scanner method came into existence.

In the bar code scanner method [2], the students were made to scan their cards against the scanner. But it was found that, this method of interpreting information from the visual information of data to be scanned had some serious drawbacks. The cards could be passed around. But it could be scanned and attendance would be marked even without the presence of the respective student. This increased the chances of proxy by a significant fraction as attendance was easily reproduced and made the whole system less secure.

In order to combat this, the biometric fingerprint system was introduced. The thumbprint of an individual is unique to every person. Marking attendance based on this would almost nullify the chances of a proxy. The Biometric finger verification [3] can be simply stated as the process of identifying match between two fingerprints to verify the identity of an individual. In this method, the students are made to scan their fingerprint and based on the match made, attendance is accordingly marked. However, this method is very costly to implement. Moreover, the change in size or form in the thumbprints of the school children would lead to the fingerprint not being properly recognized. In the longer run, it would also lead to dryness issues in children.

Another popularly implemented method is the facial recognition [4]. In this method, the live capture is compared with the stored image of a person to verify his/her identity. But this method also has certain disadvantages. The image comparison is hindered by certain facial features and this may lead to improper recognition. The information stored pertaining to images can easily be misused.

With the advent of Bluetooth technology, a tracking and monitoring system based on the same was developed [5]. In this method, tracking Bluetooth devices (fixed on walls) connected with the Bluetooth devices present in the premises. These were connected to the central Advanced RISC Machine (ARM) board which identified the person's location based on the device location. But the drawback associated with this was that the presence of Bluetooth devices was the yardstick to verify attendance and not the people themselves. This further increases chances of proxy.

Similar to Bluetooth technology, another authentication algorithm using Wireless Fidelity (Wi-Fi) module [6] was also proposed. In this method, the smart phones connected to the Wi-Fi service are scanned and checked against the registered Media Access Control (MAC) address list and 

INSTITUTIONS

phone number stored in the server. There are further chances of proxy as recorded in the Bluetooth technology. This module is highly expensive to implement as it mandates a smart phone for every student which may not be feasible.

Another popularly implemented system was the tracking system based on Global System for Mobile (GSM) [6].The GSM network communicates through relay stations and this also provided features like SMS in a much simpler fashion. But the problem associated it with it is it's inaccuracy in location determination in the densely populated areas. This may lead to incorrect marking of attendance for few students.

\section{RELATED WORKS}

There is a need to design a fully automated attendance system that replaces the previously mentioned flaws. This should also serve as a monitoring system with transparency that removes flaws like proxy. It should function as a fool proof system for an efficient and rapid management which reduces the risk of human error in attendance. Thus, the integration of Radio frequency identification (RFID) and Passive Infrared (PIR) motion sensor in an attendance system will provide an automatic system which gives better performance and efficiency [7].

RFID is a technology that makes us of radio waves to read and capture information stored in the tag of the object in which they are embedded [8]. There are two kinds of RFID tags. Active RFID tags are generally used in case of large range distances like Airports etc. Passive RFID tags are used for short field of range communication. The RFID Reader is employed to gather the information from the tag. The majority of the RFID tags contain integrated circuits compromising a transceiver.

In these kind of modules, it is preferred to use the passive RFID tag [9]. The RFID module has been implemented for attendance system extensively. The advent of this comes from the fact that RFID uses ultra-low power that does not necessitate Line of Sight operations (LOS). The RFID Reader uses an operating frequency of $125 \mathrm{kHz}$. It uses a standard RS232 serial cable or interface to plug to the desktop PC. It is fully operational with a 5V DC power supply.

Implementing a standalone RFID module would lead to the increased chances of proxy as the ID cards can be passed around to verify attendances [10]. This is very similar disadvantage associated with the barcode scanner. But, the RFID Reader module offers certain amount of advantage compared to the bar scanner that it can scan or read multiple tags at a time. Barcode scanner can read only one tag at a time. Another major advantage associated with the RFID tag is that it has relatively less cost of manufacturing. They have a longer lifetime compared to other scanners.

The PIR motion sensor is made up of piezoelectric crystal which detect the heat signature and Fresnel lens which can widen the range of a sensor. The two potentiometers are used to control the sensitivity and trigger it on time. The PIR module works on both $3.3 \mathrm{~V}$ and $5 \mathrm{~V}$. The output pin $\mathrm{D}_{\text {ou }}$ will go to a high when a person is detected and stay high as long as the person is within the range of a PIR sensor. The PIR sensors can be programmed for timeout operations. The detection distance of the PIR sensor is also programmable and can range anything up to $7 \mathrm{~m}$ in the best range cases. They can function with a working temperature of $-20^{\circ} \mathrm{C}-+80^{\circ} \mathrm{C}$.
PIR motion sensor is piezoelectric device sensitive to moving objects radiating IR [11]. PIR sensors allow to sense motion and detect whether a human has moved in or out of the sensors range. Any electrical appliance can be activated by the active presence of a human body within the coverage area. When there is no human presence, the PIR motion sensor will deactivate the electrical appliance it's embedded in.

A change in the infrared radiation causes the creation of temporary electric potential. The potential generated, would be small in amplitude and needs to be amplified considerably. This is the major reason why PIR sensors cannot be used as standalone modules. They are small, inexpensive, low-power, easy to use and don't wear out. For that reason, they are commonly found in appliances and gadgets used in homes or businesses.

\section{SYSTEM DESIGN}

In the proposed solution to the stated objective of flawless attendance marking, as described in Fig.1, we are going to integrate two technologies namely RFID and PIR motion sensing. Integrating the monitoring system with automation technology will provide a more convenient way to maintain attendance. RFID combines radio frequency and microchip technologies to create a smart system that can be used to identify, monitor, secure and do object inventory while PIR motion sensor is used to detect or count the number of objects or students.

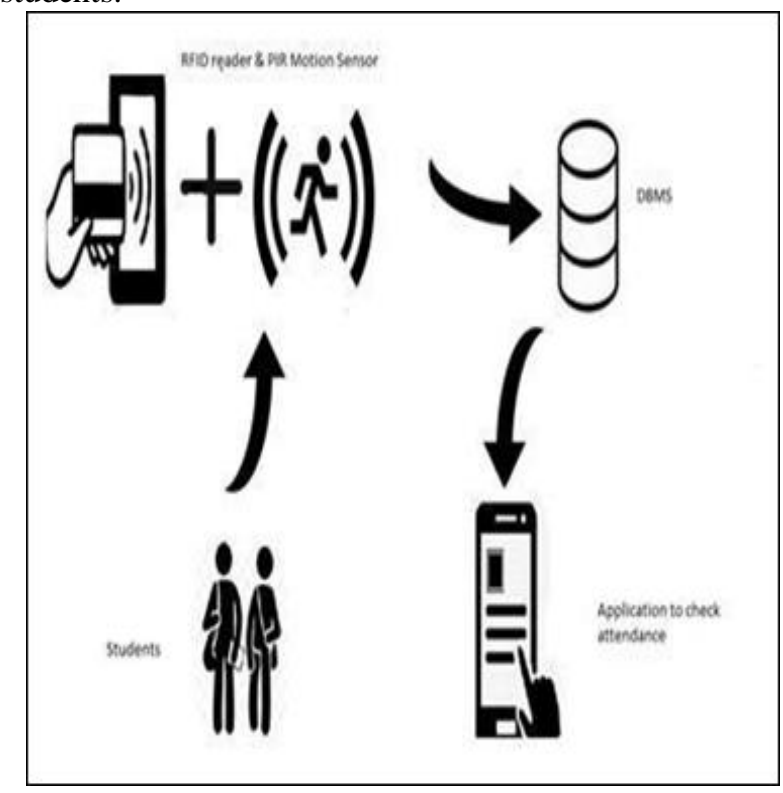

Fig. 1 System Architecture

There will be two Smart Card Readers installed in a doorway - one each for entering the doorway and another for exiting from the doorway respectively. The Smart cards distributed to students have RFID tags embedded in them. These tags contain a key value unique to every student. A user who wishes to enter or exit through the doorway has to show his/her Smart Card in front of the Smart Card Reader. The Smart Card has been configured before use and the 
required data pertaining to an individual has been electronically stored in it.

There are two PIR motion detectors used to detect the motion of the individual entering the doorway. It is placed just ahead of the doorway. As an individual enters or exits the doorway, the PIR motion sensor records his/her presence. The PIR motion sensor counts the number of users who cross through it simultaneously. It verifies the count with the number of smart tags scanned via the Smart Card Reader.

There would be a slight delay in scanning the ID card and passing through the sensor. The synchronized RFID reader and PIR Motion sensor is checked for between every lapse period. The lapse period is calculated by computing the mean of time gap taken by individuals between the RFID reader and the PIR motion sensor for a particular week and is set to a default value.

A queuing system is implemented at the doorway. The RFID and PIR modules are to be entitled i.e. synchronized to the Arduino microcontroller in order to allow entry and exit. The microcontroller is the one which performs the comparison between the two counts. If the counts are not matching, the microcontroller does not let the system respond. The queuing system opens when allowed by the microcontroller. The individual components are synchronized using middleware and controller board.

When the students pass through the queuing system, the timestamp is clogged onto the database. Similarly when the students exit through the queuing system, the exit timestamp is also noted. The difference between the entry and the exit timestamp is calculated and further checks are performed in the backend before attendance is marked for that period. This is done to ensure healthy participation for the entire period and to prevent late attendance.

The queuing system synchronization plays a crucial role in eliminating the chances of proxy. Therefore, even if a person is carrying more than one Smart Card, the person will not be allowed to enter as the smart cards scanned count and the PIR sensor count differs. Because the number of cards scanned at the RFID reader must be equal to the number of people passing through the PIR sensor to enable entry to the queuing system. This removes the malpractices like passing around Smart Cards to mark attendance.

In case, the students attempt any malpractice like proxy, it would lead to a delay in the lapse period. The lapse period has a fixed threshold value. If the delay is found to exceed the fixed threshold value, entry is restricted and attendance is not marked. The student uses his Smart Card to enter the classroom after scanning the card and passing through the PIR motion sensor. The student exits the classroom in the same procedure. database can contain whatever information is desired. In an educational Institution the details regarding student, staff such as their name, registration number etc. and other pertaining data can be stored. The database stores the attendance of the number of hours a student attended on a weekly basis. The database used in this Application (App) is Firebase which is preferred due to it's cloud services. The student can check his/her attendance percentage in the App which displays data from the Firebase. The App also provides
A database is set up to store the required information. The

an overview of the same to both the faculty and the parents of the ward.

\section{EXPERIMENTAL RESULTS}

In this model, an automated attendance system has been developed which integrates the RFID Reader and the PIR Motion Sensor. The Fig.2 illustrates a module of a PIR motion sensor and RFID Reader. They are connected and synced to an Arduino microcontroller. The Arduino microcontroller requires a $3.3 \mathrm{~V}$ or $5 \mathrm{~V}$ to function. This can be enabled by connecting the Arduino to the appropriate power supply or powering it from the connected device. In this case, it is powered from the system.

The PIR module is connected to the $5 \mathrm{~V}$ of the Arduino and RFID Reader is connected to the $3.3 \mathrm{~V}$ for the same. This is because to enable PIR to work at 3.3V, the PIR motion sensor needs to be soldered. To avoid the hassles associated with the same, it has been chosen to use these appropriate voltage power pins. They are also to be grounded via the microcontroller.

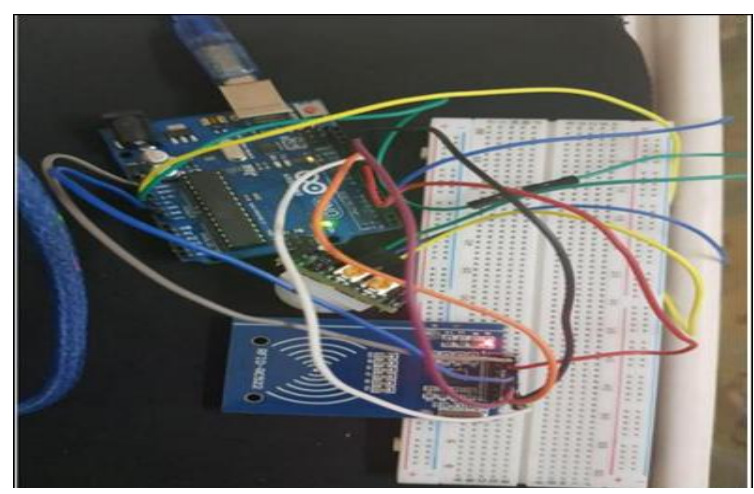

Fig. 2 Test Bed

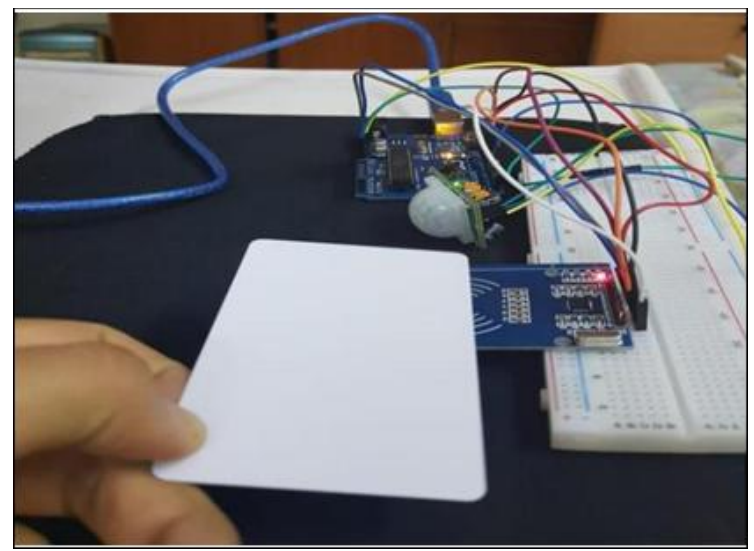

Fig. 3 Test Bed with Smart Card

Based on the results obtained from these synchronizing modules, as described in Fig. 3 and the data from the Firebase, An App has been developed using Android Studio. The application was developed in API15:Android.4.0.3 keeping in consideration the basic API version that can be enabled in all Smart phone devices. The App has been developed to serve three different stakeholders the students, the faculty and the parents respectively.

The stakeholders are provided three different portals to

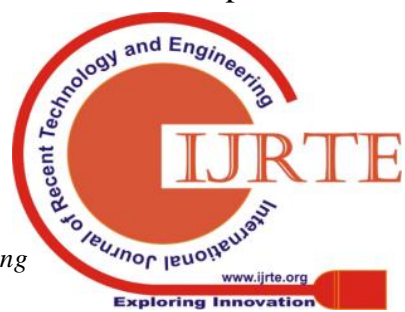




\section{RFID AND PIR MOTION SENSOR BASED AUTOMATED ATTENDANCE SYSTEM FOR EDUCATIONAL INSTITUTIONS}

access the respective information needed. The students are directed to a Login Portal, as described in Fig.4, where they are expected to enter their register number and unique student - identification code. The faculty are similarly directed to a Login Portal, as described in Fig.5, where they are expected to enter their previously configured user-name and unique faculty-identification code. As shown in Fig.6, The parents are also provided a login screen where they are expected to enter their ward's register number to check attendance status.

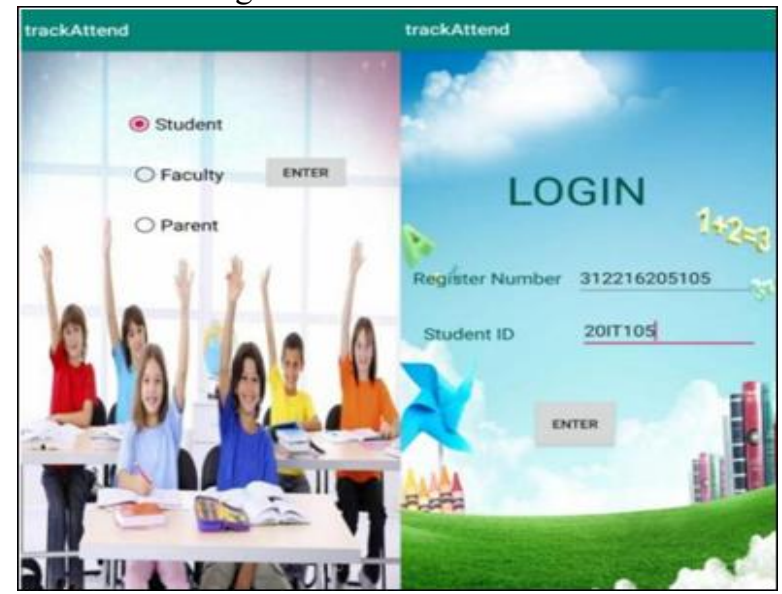

Fig. 4 Student Login Portal

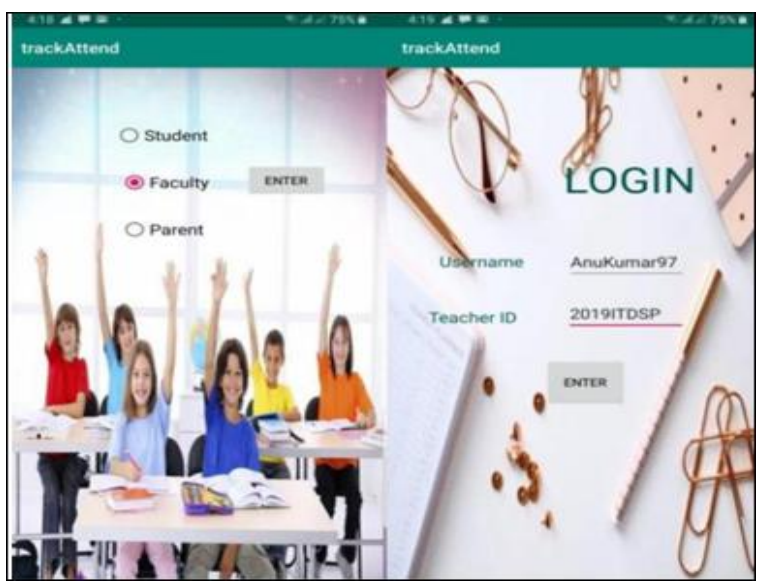

Fig. 5 Faculty Login Portal

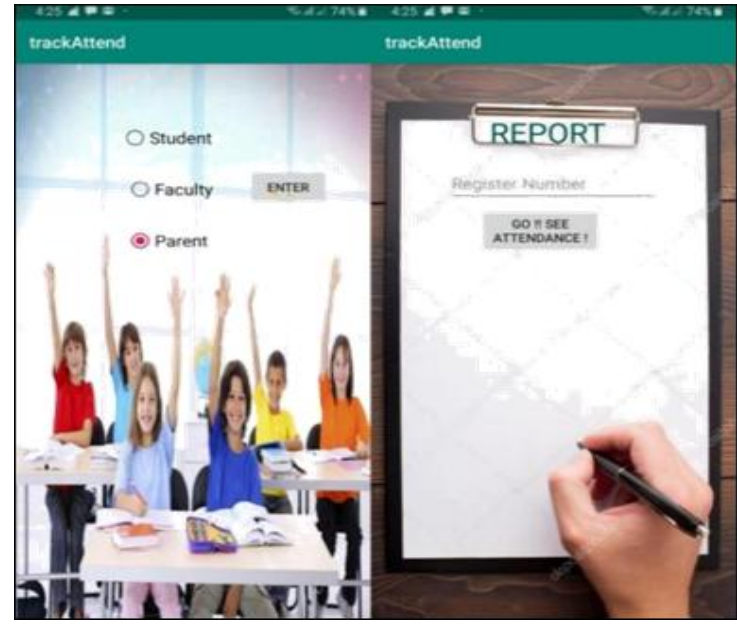

Fig. 6 Parent Login Portal

The students are allowed to access his/her monthly attendance percentage along with the weekly duration of the hours they were present for, as shown in Fig.7. It shows their overall attendance percentage.

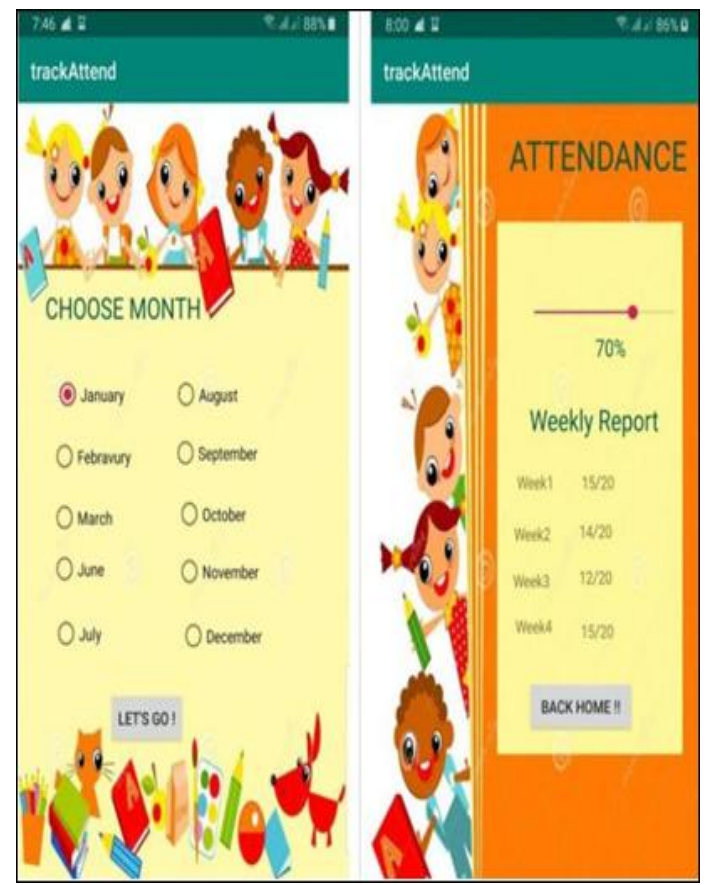

Fig. 7 Attendance Report Snapshot for Students

The teachers can assess the classroom environment by checking the fraction of students who have attendance below the threshold level, as shown in Fig.8. They can also check each student's individual attendance percentage and provide exclusive feedback.

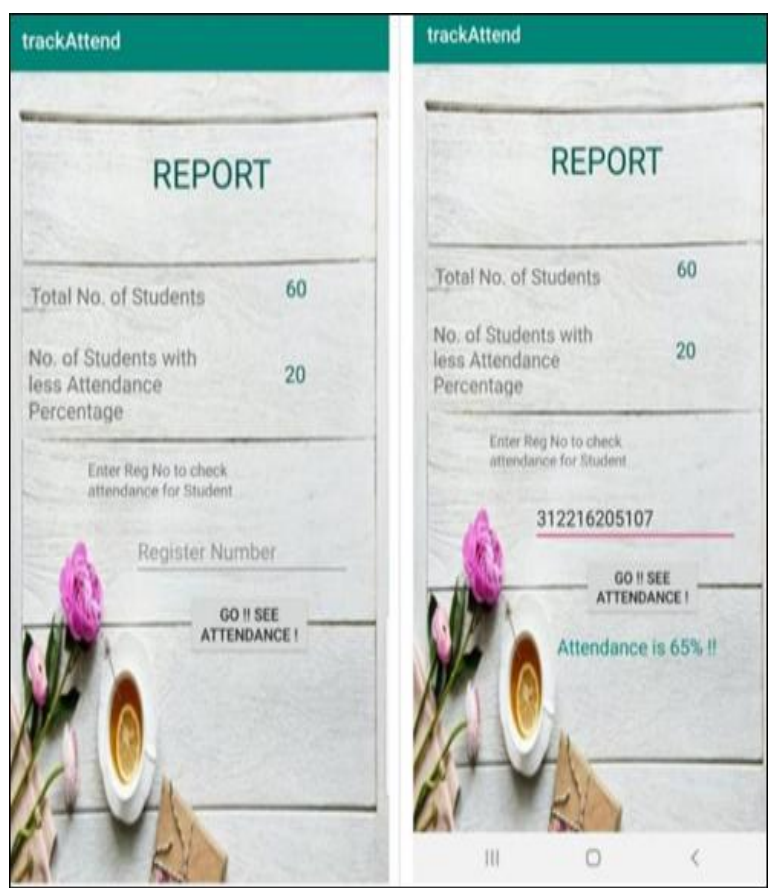

Fig. 8 Attendance Report Snapshot for Teachers

The parents can keep track of their wards by viewing the monthly attendance percentage and also checking weekly attendance status, as shown in Fig.9.

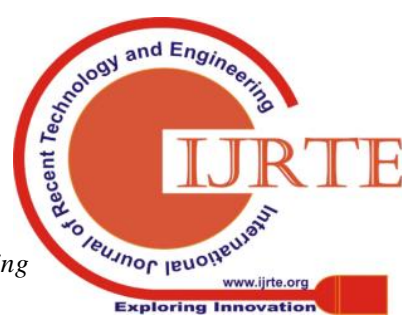




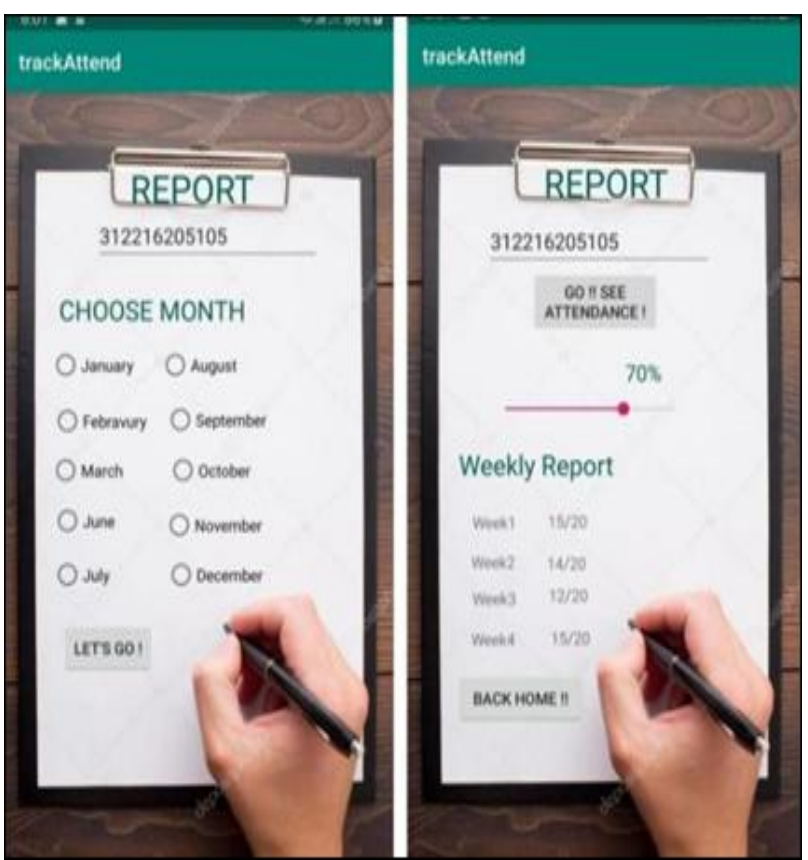

Fig. 9 Attendance Report Snapshot for Parents

\section{CONCLUSION}

Thus, this work proposed an automated attendance system for educational Institutions with the following features.

- A fully automated attendance system which ensures the efficient and ethical update of attendance in the database.

- Makes the task of attendance management easier and removes the existing malpractices.

- Allows recording of correct attendance status and making the same data available to all the stakeholders.

- A clear transparent monitoring system which ensures visibility.

- A cheap and an efficient system to improve productivity and save time.

\section{REFERENCES}

1. Monica.C,Nithya.R ,Prarthana.M, Sonika.S.V, Dr.M.Ramakrishna, "Attendance Management System" International Research Journal of Computer Science (IRJCS) ,Issue 05, Volume 4 (May 2017) .

2. Subramaniam, Hema \& Hassan, Marina \& Widyarto, Setyawan. (2013). BarCode Scanner Based Student Attendance System (SAS). TICOM (TECHNOLOGY OF INFORMATION AND COMMUNICATION). VOL. 1 NO. 3 (2013).

3. C.o, A. A.o, O. O.o, and I. E.o, "Fingerprint-Based Attendance Management System," Journal of Computer Sciences and Applications, vol. 1, no. 5, pp. 100-105, 2013.

4. S. Lukas, A. R. Mitra, R. I. Desanti, and D. Krisnadi, "Student attendance system in classroom using face recognition technique," 2016 International Conference on Information and Communication Technology Convergence (ICTC), 2016.

5. Vishal Bhalla, Tapodhan Singla, Ankit Gahlot,Vijay Gupta, "BluetoothBasedAttendanceManagement System",International Journal of Innovations in Engineering and Technology (IJIET),2013.

6. Jay Kumar,Amit Kumar,Achint Kumar Choubey,Abhinav Singh,,"Automatic Attendance Monitoring and Tracking SystemUsingBluetoothandFace Identification",International Journal of Advanced
Research in Electronics and Communication Engineering (IJARECE) Volume 5, Issue 4, April 2016.

7. D. Mahansaria, G. S. Kartik, G. Singh, H. Mishra, K. Singh, S. Sharma, and B. Amutha, "An Automated and Full-Proof Attendance Marking Scheme (TATTEND) Using Three Integrated Technologies," Communications in Computer and Information Science Recent Trends in Networks and Communications, pp. 190-199, 2010.

8. T. Lim, S. Sim, and M. Mansor, "RFID based attendance system," 2009 IEEE Symposium on Industrial Electronics Applications, 2009

9. Pushpa S. Gagare., Priyanka A. Sathe, Vedant T. Pawaskar., Sagar S. Bhave,"Smart Attendance System.",International Journal on Recent and Innovation Trends in Computing and Communication, Volume: 2 Issue: 1.

10. V.Bharani,S.Janani,Mr.A.Kanagasabapathy, "STUDENTS AUTOMATION SYSTEM USING RFID",International Journal Of Engineering And Computer Science ISSN:2319-7242,Volume 3 Issue 3 March, 2014.

11. Abhishek,Mohd Sayeed,Omer Ahmed Khan, Mohammed Owais Ahmedand Mohammed Abdul Rahman Uzair,"PIR MOTION SENSOR USING ARDUINO UNO BOARD",International conference on Science, Technology \& Management ,2018. 